\title{
Temporal association between antibiotic use and resistance in Klebsiella pneumoniae at a tertiary care hospital
}

\author{
Sukhyun Ryu ${ }^{1,2}$, Eili Y. Klein ${ }^{3,4}$ and Byung Chul Chun ${ }^{2,5^{*}}$
}

\begin{abstract}
Background: $\beta$-Lactam/ $\beta$-lactamase inhibitors (BLBLIs) were introduced into clinical practice as an alternative to carbapenems for treating multi-drug-resistant Klebsiella pneumoniae infections. However, little is known about the relationship between BLBLI treatment and antimicrobial resistance. In this study, we investigated the trends and the temporal association between antibiotic use and antimicrobial resistance in $K$. pneumoniae isolates obtained between 2012 and 2016.

Methods: Data regarding quarterly consumption (total number of prescriptions per quarter) of all BLBLIs, all thirdgeneration cephalosporins, and all fluoroquinolones at a tertiary care hospital were obtained from the Korean Health Insurance Review and Assessment Service. Susceptibility data (isolation rate of antibiotic resistance per quarter) were obtained from the existing database of the same tertiary hospital. Regression analysis was used to analyze annual trends and cross-correlations to assess the temporal association on a quarterly basis between antibiotic consumption and antibiotic resistance in K. pneumoniae.
\end{abstract}

Results: The rate of resistance to piperacillin/tazobactam in $K$. pneumoniae significantly increased over the study period $(p<0.01)$. The consumption of all BLBLIs was also found to be significantly correlated with the rate of resistance to piperacillin/tazobactam ( $\beta=0.66 ; p<0.01)$, ceftazidime $(\beta=0.54 ; p=0.02)$, and levofloxacin $(\beta=-0.60 ; p=0.01)$ with two-quarter lags. Furthermore, the consumption of all third-generation cephalosporins was significantly correlated with rates of $K$. pneumoniae resistance to ceftazidime $(\beta=0.64 ; p<0.01)$ with a twoquarter lag and levofloxacin $(\beta=0.50 ; p=0.03)$ with a quarter lag. The consumption of all fluoroquinolones correlated with resistance rates to ceftazidime $(\beta=0.14 ; p<0.01)$ with a two-quarter lag.

Conclusions: The rate of resistance to piperacillin/tazobactam in $K$. pneumoniae increased significantly over the study period and was significantly correlated with BLBLI consumption. While BLBLIs can potentially be utilized as an alternative to carbapenems, our findings reinforce concerns of resistance to these drugs. Further research is needed to understand the implications on resistance of utilizing BLBLIs as a carbapenem-sparing option.

Keywords: $\beta$-Lactamase inhibitor, Klebsiella pneumoniae, Piperacillin-tazobactam, Carbapenem resistance, Cross-correlation

\footnotetext{
* Correspondence: chun@korea.ac.kr

${ }^{2}$ Department of Epidemiology and Health Informatics, Graduate School of

Public Health, Korea University, Seoul, Republic of Korea

${ }^{5}$ Department of Preventive Medicine, Korea University College of Medicine,

73 Inchon-ro, Seongbuk-gu, Seoul 02841, Republic of Korea

Full list of author information is available at the end of the article
}

(c) The Author(s). 2018 Open Access This article is distributed under the terms of the Creative Commons Attribution 4.0 International License (http://creativecommons.org/licenses/by/4.0/), which permits unrestricted use, distribution, and reproduction in any medium, provided you give appropriate credit to the original author(s) and the source, provide a link to the Creative Commons license, and indicate if changes were made. The Creative Commons Public Domain Dedication waiver (http://creativecommons.org/publicdomain/zero/1.0/) applies to the data made available in this article, unless otherwise stated. 


\section{Background}

Klebsiella pneumoniae is a major nosocomial pathogen that causes pneumonia, urinary tract infections, and bacteremia [1]. The widespread use of broad-spectrum cephalosporins over the last several decades has resulted in extended-spectrum $\beta$-lactamase (ESBL)-producing strains of $K$. pneumoniae becoming endemic in hospitals worldwide [2, 3]. ESBLs hydrolyze $\beta$-lactam antibiotics such as penicillins and most cephalosporins, thereby conferring resistance to these drugs [4, 5]. Thus, ESBL-producing $K$. pneumoniae are usually resistant to at least one extended-spectrum cephalosporin [6]. Delays in effective treatment result in worse outcomes including increased length of hospital stays, hospitalization costs, and mortality compared to infections caused by non-ESBL producing strains [6]. The burden caused by these strains is particularly high in Korean hospitals, where approximately $53 \%$ of $K$. pneumoniae infections are caused by ESBL-producing strains [7].

To overcome this $\beta$-lactamase-mediated resistance and to enhance the efficacy of $\beta$-lactam antibiotics, $\beta$-lactam/ $\beta$-lactamase inhibitor (BLBLI) combinations such as ampicillin/sulbactam and piperacillin/tazobactams have been introduced in clinical practice [8]. Although these drugs are effective in treating infections caused by ESBL-producing organisms [8, 9], carbapenems have largely remained the treatment of choice $[2,11]$, as studies have reported conflicting results regarding the relative efficacy of therapy with BLBLIs compared to that with carbapenems [12]. However, the emergence of carbapenemase-producing Enterobacteriaceae $[13,14]$ has increased the need to utilize carbapenem-sparing treatment options, such as BLBLIs, for ESBL-related infections [10, 11]. However, it is now a growing concern that the increased use of BLBLIs will likely lead to increased resistance to these drugs $[15,16]$. One previous molecular study supports this concern, indicating that the use of BLBLIs selects for point mutants in TEM penicillinases resistant to inhibitors [17].

Although there is a generally well-established link between overall antibiotic use and resistance within hospitals $[18,19]$, information is still lacking on the relationship between BLBLI consumption and antibiotic resistance in $K$. pneumoniae. In this study, we examined the temporal association between the consumption of antibiotics commonly used in hospitals, particularly BLBLIs, third-generation cephalosporins, and fluoroquinolones, and antimicrobial resistance in K. pneumoniae. This study aimed to increase understanding of the relationship between the use of and resistance to BLBLIs to improve decision-making and policies on the use of BLBLIs as a carbapenem-sparing option.

\section{Methods}

\section{Antibiotic prescription data}

The data for antibiotic utilization (total number of antibiotics prescribed) per quarter in a designated hospital was obtained from the database of the Korean Health Insurance Review and Assessment Service (KHIRA). KHIRA covers $98 \%$ of the Korean population and includes prescription data for patients from all Korean medical institutions based on financial claims [20]. The antibiotics from the database were coded according to the World Health Organization (WHO)'s Anatomical Therapeutic Chemical (ATC) Classification [21]. Since classes of antibiotics have similar underlying mechanisms against bacteria, this classification is widely used to measure the relationship between prevalence of bacterial isolates' resistance and the exposure to different antibiotics [22, 23]. Thus, systemic antibiotic prescriptions for all BLBLIs (ATC code: J01CR), all third-generation cephalosporins (J01DD), and all fluoroquinolones (J01MA) from the hospital were included.

\section{Antimicrobial resistance data}

Microbial culture data were extracted from the existing database of the hospital and aggregated to generate quarterly estimates of antibiotic resistance [24]. The hospital has 2200 beds and has been designated as a Korean antimicrobial resistance monitoring focal point by the WHO since 1988 [25]. The specimens were collected from outpatient and inpatient departments. Identification and susceptibility testing were performed using an automated system (Vitek; bioMerieux) or disk diffusion tests in accordance with Clinical Laboratory Standards Institute (CLSI) criteria [24]. Piperacillin/tazobactam, ceftazidime, and levofloxacin were used for the susceptibility test, representing BLBLIs, third-generation cephalosporins, and fluoroquinolones, respectively. Duplicate isolates and intermediate susceptibility were not included in the resistance data [26-28]. The quarterly rate of piperacillin/tazobactam, ceftazidime, and levofloxacin resistance of $K$. pneumoniae isolates were obtained. The resistance rates were calculated as the number of resistant isolates divided by the number of tests in each quarter/year.

\section{Statistical analyses}

We used regression analysis to determine the trends of annual antibiotic prescriptions and antimicrobial resistance during the study period. $P$ values less than 0.05 with $\mathrm{R}$-squared values greater than 0.3 were considered statistically significant [29]. Furthermore, to identify the temporal relationship between the quarterly number of antibiotics prescriptions and the quarterly isolation rate of antibiotic susceptibility, a cross-correlation function test was performed. This cross-correlation function test 
is widely used to identify the time lags of one time series (antibiotic prescribing) with the possible predictors of another time series (antibiotic resistance) [30]. The Box-Jenkins method was applied to fit the time series data to seasonal autoregressive moving average models $[31,32]$. Stationary was evaluated using the augmented Dickey-Fuller test to determine whether differencing was required to rule out spurious correlations. The Akaike information criterion test and the portmanteau test of the residuals were used to identify the best model fit. The residuals from each time series model were used to evaluate the temporal relationship between antibiotics prescription and isolation rate of $K$. pneumoniae with antibiotic resistance [33]. All statistical analyses were performed using $\mathrm{R}$ version 3.2.4 ( $\mathrm{R}$ Foundation for Statistical Computing, Vienna, Austria).

\section{Results}

The average annual number of antibiotics prescriptions during the study period was 69,100 (range 27,37184,128 ) for all BLBLIs; 53,300 (range 22,789-70,514) for all third-generation cephalosporins; and 28,536 (range 12,324-35,368) for all fluoroquinolones (Table 1). There was no significant trend in prescribing rates over the study period.

The yearly numbers of $K$. pneumoniae isolates from 2012 to 2016 for this analysis were 1524, 1609, 1585, and 1754 . The mean rates of $K$. pneumoniae resistance to piperacillin/tazobactam, ceftazidime, and levofloxacin were $41 \%$ (range $22-49$ ), 34\% (range 14-44), and 30\% (range 23-44), respectively (Table 2). There was a stable trend in resistance rates against ceftazidime and levofloxacin; however, the resistance rate against piperacillin/tazobactam significantly increased from $34 \%$ (range: $22-46)$ in 2012 to $46 \%$ (range: 43-49) in 2016; $(p<0.01)$.

In a bivariate analysis, the lagged (two quarters) quarterly number of all BLBLI prescriptions significantly correlated with $K$. pneumoniae resistance to piperacillin/tazobactam $(\beta=0.66 ; p<0.01)$, ceftazidime $(\beta=0.54 ; p=0.02)$, and levofloxacin $(\beta=-0.60 ; p=0.01)$. Similarly, the lagged quarterly number of all third-generation cephalosporin prescriptions significantly correlated with $K$. pneumoniae resistance to ceftazidime ( $\beta=0.64 ; p<0.01$; two quarters) and levofloxacin $(\beta=0.50 ; p=0.03$; one quarter). The lagged quarterly number of all quinolone prescriptions correlated with $K$. pneumoniae resistance to ceftazidime ( $\beta=0.14 ; p<0.01$; two quarters), although there was no significant association with levofloxacin resistance $(\beta=0.23 ; p=0.35)$ (Table 3$)$.

\section{Discussion}

The emergence of ESBL-producing $K$. pneumoniae strains and the increasing rates of carbapenem resistance have reduced therapeutic options for infections caused by these organisms $[2,6,10]$. BLBLIs such as piperacillin/tazobactam have been suggested as alternatives to carbapenems $[10,11,12]$ and may be comparable in efficacy to carbapenems against ESBL-producing $K$. pneumoniae infections [34]. However, although the evidence for their use as an alternative to carbapenems has been conflicting [12], their use has been increasing. This has led to increasing concern about resistance, which undermines the efficacy of these drugs [12].

To improve policies around the potential replacement of carbapenems with BLBLIs, it is important to examine the relationship between BLBLI use and antimicrobial resistance using a seasonal analysis, which previous studies used to demonstrate the linkage between seasonality and antibiotic use [22, 35].

The greatest positive temporal association was found between all BLBLI utilization and piperacillin/tazobactam resistance in K. pneumoniae. However, no significant correlation was found for the use of the third-generation cephalosporins and fluoroquinolone. This was presumably due to the selection pressure of antibiotic use on the prevalence of resistant isolates. This finding is consistent with results of a previous study that identified a significant correlation between piperacillin/tazobactam use and subsequent piperacillin/tazobactam resistance in Enterobacteriaceae [36].

We also found that ceftazidime resistance in K. pneumoniae temporally correlated with prescriptions for all BLBLIs, all third-generation cephalosporins, and all fluoroquinolones. Consistent with our findings, a previous report indicated that the presence of ceftazidime-resistant Klebsiella spp. is significantly positively associated with

Table 1 Average quarterly number of antibiotic prescriptions in a tertiary hospital from 2012 to 2016

\begin{tabular}{|c|c|c|c|c|c|}
\hline \multirow[t]{2}{*}{ Antibiotics } & \multicolumn{5}{|c|}{ Annual mean number of antibiotics prescriptions } \\
\hline & 2012 & 2013 & 2014 & 2015 & 2016 \\
\hline$\beta$-lactam/ $\beta$-lactamase inhibitors & $\begin{array}{l}68,907 \\
(53,899-80,221)\end{array}$ & $\begin{array}{l}72,725 \\
(63,235-84,128)\end{array}$ & $\begin{array}{l}71,561 \\
(68,880-76,537)\end{array}$ & $\begin{array}{l}63,638 \\
(27,371-80,928)\end{array}$ & $\begin{array}{l}68,512 \\
(64,378-71,719)\end{array}$ \\
\hline Third-generation cephalosporins & $\begin{array}{l}63,304 \\
(60,238-70,514)\end{array}$ & $\begin{array}{l}64,784 \\
(60,468-67,074)\end{array}$ & $\begin{array}{l}51,578 \\
(44,996-54,267)\end{array}$ & $\begin{array}{l}39,007 \\
(22,789-46,250)\end{array}$ & $\begin{array}{l}45,999 \\
(44,346-47,384)\end{array}$ \\
\hline Fluoroquinolones & $\begin{array}{l}29,300 \\
(26,233-33,663)\end{array}$ & $\begin{array}{l}31,621 \\
(28,920-35,297)\end{array}$ & $\begin{array}{l}27,582 \\
(21,069-35,286)\end{array}$ & $\begin{array}{l}25,900 \\
(12,324-32,129)\end{array}$ & $\begin{array}{l}28,195 \\
(19,737-35,368)\end{array}$ \\
\hline
\end{tabular}


Table 2 Quarterly antimicrobial resistance in K. pneumoniae in a tertiary hospital from 2012 to 2016

\begin{tabular}{|c|c|c|c|c|c|}
\hline \multirow[t]{2}{*}{ Antibiotics } & \multicolumn{5}{|c|}{ Isolation rate (\%) of antibiotic-resistant Klebsiella pneumoniae } \\
\hline & 2012 & 2013 & 2014 & 2015 & 2016 \\
\hline Piperacillin/Tazobactam & $\begin{array}{l}33.75 \\
(22.00-46.00)\end{array}$ & $\begin{array}{l}42.50 \\
(40.00-45.00)\end{array}$ & $\begin{array}{l}41.00 \\
(39.00-45.00)\end{array}$ & $\begin{array}{l}40.25 \\
(30.00-46.00)\end{array}$ & $\begin{array}{l}46.00 \\
(43.00-49.00)\end{array}$ \\
\hline Ceftazidime & $\begin{array}{l}40.75 \\
(38.00-44.00)\end{array}$ & $\begin{array}{l}38.50 \\
(36.00-42.00)\end{array}$ & $\begin{array}{l}28.75 \\
(26.00-31.00)\end{array}$ & $\begin{array}{l}27.00 \\
(14.00-35.00)\end{array}$ & $\begin{array}{l}36.00 \\
(33.00-38.00)\end{array}$ \\
\hline Levofloxacin & $\begin{array}{l}28.50 \\
(27.00-32.00)\end{array}$ & $\begin{array}{l}29.00 \\
(27.00-31.00)\end{array}$ & $\begin{array}{l}26.25 \\
(23.00-30.00)\end{array}$ & $\begin{array}{l}32.50 \\
(27.00-44.00)\end{array}$ & $\begin{array}{l}33.00 \\
(30.00-36.00)\end{array}$ \\
\hline
\end{tabular}

Data indicate mean number of prescriptions (Min-Max)

cephalosporin and fluoroquinolone use at the hospital level [37]. Regarding the positive association between fluoroquinolone use and the prevalence of ceftazidime resistance to K. pneumoniae, this may be explained by the transferrable plasmid (qnr gene) among ESBL-producing strains which leads to quinolone resistance [38]. Thus, these correlations are likely due to the ability of ESBLs to confer resistance to multiple classes of antibiotics [39].

However, we did not find an association between levofloxacin resistance in K. pneumoniae and the consumption of quinolones; this result was in contrast to some previous reports which found that increasing fluoroquinolone use was associated with rising incidence of quinolone resistance in Enterobacteriaceae [40, 41]. Additionally, we found that levofloxacin resistance in K. pneumoniae was negatively associated with all BLBLI utilization and positively associated with third-generation cephalosporin utilization. These results may be due to the low level of fluctuation from the quarterly number of fluoroquinolones used, and the low magnitude of utilization likely affected the resistance emergence rates [42].

This is the first study to describe the temporal relationship between BLBLI use and the resistance to them in $K$. pneumoniae in a tertiary level hospital. The strong correlations reported in our study suggest that replacing carbapenems with BLBLIs is not an ideal long-term solution, as resistance is likely to spread rapidly. A previous study in Korea showed that broad-spectrum

Table 3 Cross-correlation coefficients between quarterly antibiotics prescriptions and quarterly isolation rates of antibiotic-resistant K pneumoniae from 2012 to 2016

\begin{tabular}{llll}
\hline Antibiotics & \multicolumn{3}{l}{ Antibiotic-resistant Klebsiella pneumoniae } \\
\cline { 2 - 4 } & $\begin{array}{l}\text { Piperacillin/ } \\
\text { tazobactam }\end{array}$ & Ceftazidime & Levofloxacin \\
& 0.66 & 0.54 & -0.60 \\
B-lactam/B-lactamase & $p<0.01$ & $p=0.02$ & $p=0.01$ \\
inhibitors & 2 quarters lag & 2 quarters lag & 2 quarters lag \\
& -0.11 & 0.64 & 0.50 \\
Third-generation & $p=0.65$ & $p<0.01$ & $p=0.03$ \\
cephalosporins & - & 2 quarters lag & 1 quarter lag \\
& 0.26 & 0.14 & 0.23 \\
Fluoroquinolones & $p=0.29$ & $p<0.01$ & $p=0.35$ \\
& - & 2 quarters lag & - \\
\hline
\end{tabular}

penicillins including BLBLIs were more frequently prescribed than narrow-spectrum penicillins [43]. Another study demonstrated that this is presumably due to the physician's desire to avoid missing an unlikely or unidentified resistant pathogen [44]. Thus, BLBLIs should be suggested to be used with caution as a carbapenem-sparing option. Further research is needed to evaluate the impact of antimicrobial stewardship programs to balance the use and resistance across drug classes.

Although the results from our study were significant, there are some limitations. First, the study was ecological in nature and may not represent biological associations at the individual level. However, ecological studies are often used to suggest the relationship between use of an antibiotic and resistance to that antibiotic on population level $[35,45]$. Second, we used the total number of antibiotics prescriptions as a measure of antibiotic consumption instead of using defined daily doses (DDDs) in this study. However, a previous study indicated that evaluating the total number of antibiotics prescriptions is a useful method to measure the strength of the relationship between antibiotic consumption and antibiotic resistance [41], and DDDs are typically highly correlated with the total number of prescriptions. Third, other factors such as the inconsistency of the patient population, transferred patients having resistant organisms from different hospitals, possible epidemics, and infection control measures during the study the period may have affected the level of resistance in our findings.

\section{Conclusions}

In this study, we identified a significant temporal correlation between BLBLI use and piperacillin/tazobactam resistance in $K$. pneumoniae. The increasing trend in the rates of resistance to piperacillin/tazobactam in $K$. pneumoniae observed in this study raises concerns regarding the appropriate use of BLBLIs as carbapenem-sparing antibiotics. Our results suggest that new interventions aimed at reducing the use of BLBLIs may help retain their efficacy as a carbapenem-sparing alternative. 


\section{Abbreviations}

BLBLIs: $\beta$-lactam/ $\beta$-lactamase inhibitors; CLSI: Clinical Laboratory Standards Institute; DDDs: Defined daily doses; ESBLs: Extended-spectrum $\beta$-lactamases; KHIRA: Korean Health Insurance Review and Assessment Service; WHO: World Health Organization

\section{Funding}

This work was supported by the Research Institute for Healthcare Policy, KMA in 2016.

The funding agency had no role in the design of the study and collection, analysis, and interpretation of data, and in writing the manuscript.

\section{Authors' contributions}

SR was responsible for the design of the study and collected the data. SR EIK, and CBC analyzed the data. SR and EIK prepared the initial and revised drafts of the manuscript. SR and CBC were responsible for validation, analysis, and interpretation of the data. All authors contributed to the final version of the manuscript. All authors read and approved the final manuscript.

\section{Author's information}

SR is a field epidemiologist at the Gyeonggi Provincial Government, Republic of Korea. His areas of interests include infectious disease epidemiology and antimicrobial resistance.

\section{Ethics approval and consent to participate}

This study was exempt from ethical approval from the Institutional Review Board designated by the Korean Ministry of Health and Welfare: P01-201710-21-007.

\section{Consent for publication}

Not applicable.

\section{Competing interests}

The authors report no conflicts of interest relevant to this article.

\section{Publisher's Note}

Springer Nature remains neutral with regard to jurisdictional claims in published maps and institutional affiliations.

\section{Author details}

'Division of Infectious Disease Control, Gyeonggi Provincial Government, Suwon, Republic of Korea. ${ }^{2}$ Department of Epidemiology and Health Informatics, Graduate School of Public Health, Korea University, Seoul, Republic of Korea. ${ }^{3}$ Center for Disease Dynamics, Economics \& Policy, Washington, D.C, USA. ${ }^{4}$ Department of Emergency Medicine, Johns Hopkins University, Baltimore, USA. ${ }^{5}$ Department of Preventive Medicine, Korea University College of Medicine, 73 Inchon-ro, Seongbuk-gu, Seoul 02841, Republic of Korea.

\section{Received: 2 August 2017 Accepted: 4 July 2018}

\section{Published online: 16 July 2018}

\section{References}

1. Tsay RW, Siu LK, Fung CP, Chang FY. Characteristics of bacteremia between community-acquired and nosocomial Klebsiella pneumoniae infection: risk factors for mortality and the impact of capsular serotypes as a herald for community-acquired infection. Arch Intern Med. 2002; 162:1021-7.

2. Paterson DL, Bonomo RA. Extended-spectrum beta-lactamases: a clinical update. Clin Microbiol Rev. 2005;18:657-86.

3. Kang Cl, Kim SH, Kim DM, Park WB, Lee KD, Kim HB, et al. Risk factors for and clinical outcomes of bloodstream infections caused by extendedspectrum beta-lactamase-producing Klebsiella Pneumoniae. Infect Control Hosp Epidemiol. 2004;25:860-7.

4. Bradford PA. Extended spectrum- $\beta$-lactamases in the 21 st century: characterization, epidemiology, and detection of this important resistance threat. Clin Microbiol Rev. 2001;14:933-51.

5. Peterson DL, Ko WC, Gottberg AV, Mohapatra S. Antibiotic therapy for Klebsiella pneumonia bacteremia: implications of production of extendedspectrum $\beta$-lactamases. Clin Infect Dis. 2003;39:31-7.
6. Stone PW, Gupta A, Loughrey M, Della-Latta P, Cimiotti J, Larson E, et al Attributable costs and length of stay of an extended-spectrum betalactamase-producing Klebsiella pneumoniae outbreak in a neonatal intensive care unit. Infect Control Hosp Epidemiol. 2003;25:601-6.

7. Kim HS, Kim Jl, Chan C. Monitoring of antimicrobial resistance on non-tertiary hospitals in Korea, 2007-2013. Public Health Wkly Rep. 2015:8:1092-5.

8. Drawz SM, Bonomo RA. Three decades of $\beta$-lactamase inhibitors. Clin Microbiol Rev. 2010;23:160-201.

9. Piroth L, Aube H, Doise JM, Vincent-Martin M. Spread of extended-spectrum $\beta$-lactamase-producing Klebsiella pneumonia: are $\beta$-lactamase inhibitors of therapeutic value? Clin Infect Dis. 1998;27:76-80.

10. Harris PNA, Yin M, Jureen R, Chew J, Ali J, Paynter S, et al. Comparable outcomes for $\beta$-lactam/ $\beta$-lactamase inhibitor combinations and carbapenems in definitive treatment of bloodstream infections caused by cefotaxime-resistant Escherichia coli or Klebsiella pneumoniae. Antimicrob Resist Infect Control. 2015:4:14

11. Vardakas KZ, Tansarli GS, Rafailidis PI, Falagas ME. Carbapenems versus alternative antibiotics for the treatment of bacteremia due to Enterobacteriaceae producing extended-spectrum $\beta$-lactamases: a systematic review and meta-analysis. J Antimicrob Chemother. 2012;67:2793-803.

12. Tamma PD, Rodriguez-Bano J. The use of non-carbapenem, $\beta$-lactams for the treatment of extended-spectrum $\beta$-lactamase infections. Clin Infect Dis. 2017;64:972-80.

13. Pletz MW, Rau M, Bulitta J, De Roux A, Burkhardt O, Kruse G, et al. Ertapenem pharmacokinetics and impact on intestinal microflora, in comparison of ceftriaxone, after multiple dosing in male and female volunteers. Antimicrob Agents Chemother. 2004;48:3765-72.

14. Canton R, Akova M, Carmeli Y, Giske CG, Glupczynski U, Gniadkowski M, et al. Rapid evolution and spread of carbapenemases among Enterobacteriaceae in Europe. Clin Microbiol Infect. 2012;18:413-31.

15. Hoban DJ, Bouchillon SK, Hawser SP, Badal RE, LaBombardi VJ, DiPersio J. Susceptibility of gram-negative pathogens isolated from patients with complicated intra-abdominal infections in the United States, 2007-2008: results of the study for monitoring antimicrobial resistance trends (SMART). J Antimicrob Chemother. 2010;54:3031-4.

16. Hsueh PR, Badal RE, Hawser SP, Hoban DJ, Bouchillon SK, Ni Y, et al. Epidemiology and antimicrobial susceptibility profiles of aerobic and facultative gram-negative bacilli isolated from patients with intra-abdominal infections in the Asia-Pacific region: 2008 results from SMART (study for monitoring antimicrobial resistance trends). Int J Antimicrob Agents. 2010; 36:408-14.

17. Belaaouaj A, Lapoumeroulie C, Canica MM, Vedel G, Nevot P, Krishnamoorthy $R$, et al. Nucleotide sequences of the genes coding for the TEM-like betalactamases IRT-1 and IRT-2 (formerly called TRI-1 and TRI-2). FEMS Microbiol Lett. 1994;120:75-80.

18. Hsueh PR, Chen WH, Luh KT. Relationships between antimicrobial use and antimicrobial resistance in gram-negative bacteria causing nosocomial infections from 1991-2003 at a university hospital in Taiwan. Int J Antimicrob Agents. 2005:26:463-72.

19. Xu J, Duan X, Wu H, Qi Z. Surveillance and correlation of antimicrobial usage and resistance of pseudomonas aeruginosa: a hospital populationbased study. PLoS One. 2013;8:e78604.

20. Kim L, Kim JA, Kim S. A guide for the utilization of health insurance review and assessment service national patient sample. Epidemiol Health. 2014;36: e2014008.

21. World Health Organization. 2016. WHO Collaborating Centre for Drug Statistics Methodology. Structure and principles. http://www.whocc.no/atc/ structure_and_principles. Accessed 2 Aug 2017.

22. Goossens H, Ferech M, Stichele RV, Elseviers M. Outpatient antibiotic use in Europe and association with resistance: a cross-national database study. Lancet. 2005;365:579-87.

23. Goossens H. Antibiotic consumption and link to resistance. Clin Microbiol Infect. 2009;15:12-5.

24. Antimicrobial Resistance Newsletter. Research Institute of Bacterial Resistance and Department of Laboratory Medicine, Severance Hospital, Seoul. Republic of Korea.

25. Research Institution of Bacterial Resistance. http://www.whonetkorea.org. Accessed 2 Aug 2017.

26. Clinical and Laboratory Standard Institute. Performance standards for antimicrobial susceptibility testing: 19th informational supplement M100-S19. Wane: Clinical and Laboratory Standard Institute; 2009. 
27. Clinical and Laboratory Standard Institute. Performance standards for antimicrobial susceptibility disk susceptibility test; Approved standard. 11th ed. Wane: Clinical and Laboratory Standards Institute; 2012.

28. Clinical and Laboratory Standard Institute. Performance standards for antimicrobial susceptibility testing: 23 rd informational supplement. Wane: Clinical and Laboratory Standard Institute; 2013.

29. Hsu LY, Tan TY, Tam VH, Kwa A, Fisher DA, Koh TH. Network for antimicrobial resistance surveillance. Surveillance and correlation of antibiotic prescription and resistance of gram-negative bacteria in Singaporean hospitals. Antimicrob Agents Chemother. 2010;5:1173-8.

30. Gilca R, Fortin E, Frenettte C, Longtin Y, Gourdeau M. Seasonal variation in Clostridium difficile infections are associated with influenza and respiratory syncytial virus activity independently of antibiotic prescriptions: a time series analysis in Quebec, Canada. J Antimicrob Chemother. 2012;56:639-46.

31. Cowperwait PSP, Metcalfe AV. Introductory time series with R. New York: Springer; 2009.

32. Box GEP, Jenkins GM, Reinsel GC. Time series analysis: forecasting and control, $3^{\text {rd }}$ ed. Englewood cliffs, NJ: prentice hall, 1994. Helfenstein U. Box-Jenkins modelling in medical research. Stat Methods Med Res. 1996;5:3-22.

33. Hyndman RJ, Athanasopoulos G. Forecasting; principles and practice. New York: O Texts; 2014.

34. Ng TM, Khong WX, Harris PNA, De PP, Chow A, Tambyah PA, et al. Empiric piperacillin-Tazobactam versus Carbapenems in the treatment of Bacteraemia due to extended- Spectrum Beta-lactamase-producing Enterobacteriaceae. PLoS One. 2016;11:e0153696.

35. Sun L, Klein EY, Laxminarayan R. Seasonality and temporal correlation between community antibiotic use and resistance in the United States. Clin Infect Dis. 2012;7:1-8.

36. Lai CC, Wang CY, Chu CC, Tan CK, Lu CL, Lee YC, et al. Correlation between antibiotic consumption and resistance of gram-negative bacteria causing healthcare-associated infections at a university hospital in Taiwan from 2000 to 2009. J Antimicrob Chemother. 2011;66:1374-82.

37. Jacoby TS, Kuchenbeckers RS, Dos Santos RP, Magedanz L, Guzatto P, Moreira LB. Impact of hospital-wide infection rate, invasive procedures use and antimicrobial consumption on bacterial resistance inside an intensive care unit. J Hosp Infect. 2010;75:23-7.

38. Wang M, Sahm DF, Jacoby GA, Hooper DC. Emerging plasmid-mediated quinolone resistance associated with the anr gene in Klebsiella pneumoniae clinical isolates in the United States. Antimicrob Agents Chemother. 2004;48: $1295-9$.

39. Liu X, Thungrat K, Boothe DM. Occurrence of OXA-48 carbapenemase and other $\beta$-lactamase genes in ESBL-producing multidrug resistant Escherichia coli from dogs and cats in the United States, 2009-2013. Front Microbiol. 2016;: :1057.

40. Livermore DM, James D, Reacher M, Graham C, Nichols T, Stephens P, et al. Trends in fluoroquinolone (ciprofloxacin) resistance in Enterobacteriaceae from Bacteremias, England and Wales, 1990-1999. Emerg Infect Dis. 2002;8: 473-8.

41. Bolon MK, Wright SB, Gold HS, Carmeli Y. The magnitude of the association between fluoroquinolone use and quinolone-resistant Escherichia coli and Klebsiella pneumoniae may be lower than previously reported. Antimicrob Agents Chemother. 2004;48:1934-40.

42. Peterson LR. Squeezing the antibiotic balloon: the impact of antimicrobial classes on emerging resistance. Clin Microbiol Infect. 2005;11:4-16.

43. Yoon YK, Park GC, An H, Chun BC, Sohn JW, Kim MJ. Trends of antibiotic consumption in Korea according to national reimbursement data (2008-2012): a population-based epidemiologic study. Medicine. 2015;94:e2100.

44. Livorsi D, Comer A, Matthias MS, Perencevich EN, Bair MJ. Factors influencing antibiotic-prescribing decisions among inpatient physicians: a qualitative investigation. Infect Control Hosp Epidemiol. 2015;36:1065-72.

45. Iosifidis E, Antachopoulos C, Tsivitanidou M, Katragkou A, Farmaki E, Tsiakou $M$, et al. Differential correlation between rates of antimicrobial drug consumption and prevalence of antimicrobial resistance in a tertiary care hospital in Greece. Infect Control Hosp Epidemiol. 2008;29:615-22.

\section{Ready to submit your research? Choose BMC and benefit from:}

- fast, convenient online submission

- thorough peer review by experienced researchers in your field

- rapid publication on acceptance

- support for research data, including large and complex data types

- gold Open Access which fosters wider collaboration and increased citations

- maximum visibility for your research: over $100 \mathrm{M}$ website views per year

At BMC, research is always in progress.

Learn more biomedcentral.com/submissions 